\title{
Prevalence of Bovine Trypanosomosis in and around Nekemte Areas, East Wollega Zone, Ethiopia
}

\author{
Feyera Gemeda \\ School of Veterinary Medicine, Jimma University, Jimma, Ethiopia \\ Email: qafayera.game@gmail.com
}

Received 7 May 2015; accepted 24 May 2015; published 29 May 2015

Copyright (C) 2015 by author and OALib.

This work is licensed under the Creative Commons Attribution International License (CC BY). http://creativecommons.org/licenses/by/4.0/

(c) $\underset{\mathrm{EY}}{\mathrm{i}}$ Open Access

\begin{abstract}
A cross-sectional study was conducted from November 2010 to April 2011 to determine the prevalence of trypanosomosis and to identify the prevailing species of trypanosomes in cattle present in and around Nekemte. Blood sample were collected from ear vein of 400 cattle and then examined using thin and thick smear method followed by Buffy coat examination. Anemic status was determined by Packed Cell Volume (PCV). Out of 400 samples were examined, 36 (9\%) were positive, out of which $26(0.065 \%)$ had Trypanosoma vivax and $10(0.025 \%)$ had $T$. congolense. The mean PCV of the infected animal is $\mathbf{1 9 . 3 6}$ and that of non infected animal is 27.54, which indicates a significant difference between these animals. Trypanosomosis is a fatal and economically devastating disease and the major constraint to production by causing loss of the livestock. Therefore, the better strategies to prevent this disease includes: avoidance of animals from tsetse-infested areas, tsetse fly control by using different scientific methods (such as, sterile insect technique, use of accaricides), prophylactic use of trypanocidal drugs, keeping of trypanoresistant breeds and good husbandry practice for the prevention of the disease.
\end{abstract}

\section{Keywords}

Prevalence, Trypanosomosis, Bovine, East Wollega Zone, Nekemte Town, T. vivax, T. congolense

Subject Areas: Food Science \& Technology, Public Health

\section{Introduction}

African continent is faced with the challenge of satisfying dramatic increase in demand for livestock products, especially milk and meat [1]. This is also a problem in countries like Ethiopia in general and in and around Nekemte areas in particular. Domesticated species play an important role in supporting human populations and 
in generating income and economic activity [2]. Large areas of natural grassland could be exploited better to support the increasing demand for livestock products and their highest contributions to market economies understood. Animal diseases, especially those caused by parasites, are major constraints to production in these areas and trypanosomosis is arguably the most important one of these [3]. Its transmission involves tsetse fly (Glossina) species. It can also be transmitted both mechanically and excrementally. As a result of trypanosomes threat, large proportion of livestock population is found in the high lands that are assumed to be tsetse-free [4]. Although livestock trypanosomes are a well-known constraint to livestock production in Ethiopia, title attention has been paid to the trypanosomes situation. Nevertheless, livesstock are backbone of the rural economy in this region where diverse species of animals including cattle, sheep and goat are found. To clarity bovine trypanosomes situation, a survey was conducted to determine the prevalence of trypanosomes infections in East Wollega from November 2010 to April 2011. A total of 400 animals were investigated, and 9\% were infected with trypanosomes.

With the exception of T. equiperdum of equines which is a venereal disease, all other species of trypanosomes have arthropod vectors in which the transmission occurs either cyclically or non cyclically. In cyclical transmission the arthropods are the necessary intermediate in which the trypanosomes multiply, undergoing a serious of morphological transformations before form infective for mammalian host produced [5]. When multiplication occurs in digestive tract and proboscis, metatrypanosome is transmitted when feeding, the process is known as anterior station development and various species of trypanosomes which use this process are often considered as a group called salivaria and are all transmitted by tsetse flies [6]. In other trypanosomes, multiplication and transformation occurs in the gut and the infective forms migrate to the rectum and are passed with the faeces. This is a posterior station development and the trypanosomes species are grouped together as stercoraria. In domestic animals, there are a relatively non pathogenic trypanosomes; such as T. theileri, and T. mellophagium transmitted by tabanid flies and sheep ked respectively, but this is not the cause in man in which $T$. cruzi, the cause of the serious Chagas disease in south America, is transmitted in feces of ruduviid bugs [7].

In ruminants the major signs are anemia, generalized enlargement of the superficial lymph glands, lethargy and progressive loss of body condition. Fever and loss of appetite occur intermittently during parasitemic peaks, the latter becoming marked in the terminal stages of the disease. Typically, the disease is chronic extending over several months and usually terminates fatally if untreated. As a herd phenomenon, the growth of young is stunted, while adults show decreased fertility, and if pregnant, may abort. In terminal stages animal become extremely weak, the lymph nodes are reduced in size and there is often a jugular pulse. Death is also associated with congestive heart failure due to anemia and myocarditis. Occasionally, with some strains of $T$. vivax, the disease is acute, death occurring within 2 - 3 weeks of infection preceded by fever, anemia and wide spread hemorrhages [8].

In horses T. brucie infections may be acute or chronic, often accompanied by oedema of the limbs and genitalia. In the pig, $T$. congolense infections are mild and chronic in contrast to those associated with $T$. simiae where the disease is hyperacute and death occurs within a day. Dog and cat are susceptible to T. brucei and T. congolense. The disease is usually acute and apart from signs of fever, anemia, myocarditis, corneal opacity is often a feature. Presence of large number of trypanosomes causes the temperature over $40^{\circ} \mathrm{C}$. Since parasitaemic animals survive for prolonged periods; there are ample opportunities for fly transmission, especially of $T$. brucei and $T$. congolense. In contrast, some strains of $T$. vivax in cattle and $T$. simaei in domestic pigs kill their hosts within 1 - 2 weeks so that chances of fly transmission are more limited [9].

Disorders of the nervous system vary in nature and dominate the second stage disease development in man. There are neurological changes resulting in aggressive signs, ataxia and convulsions. The fist symptoms are irritability, hyperesthesia, and insomnia, leading to somnolence, coma and paralysis [10]. Metacyclicand blood stream trypanosomes posses a glycoprotein coat, which is antigenic and provokes the formation of antibodies and this causes opsonization, and lysis of trypanosomes. Unfortunately by the time antibody is produced, a proportion of trypanosomes have altered the chemical composition of their glycoprotein coat and then displayed a different antigenic surface, and they were unaffected by antibody [11]. Those trypanosomes possessing this new variant antigen multiply to produce a second wave of parasitemia; the host produce a second antibody, but again the glycoprotein coat has altered in number of trypanosomes so that a third wave of parasitemia occurs. This process of antigenic variation is associated with waves and remission of parasitemias, and is often at weekly intervals or may continue for months, usually with fatal outcome [12].

T. evansi causes a disease called sura in camels while T. equiperdum causes dourine in horses and donkeys which is transmitted through coitus. Pathogenic trypanosomes differ widely in virulence and are apparently non 
pathogenic in mammalian reservoir hosts [13]. Most of them are parasites of variety of domestic and wild animals, in some of which they produce a fulminating disease, in some chronic disease and in others mild or inapparent infections. Strains with the species of parasite also differ greatly in virulence, some strains produce rapidly fatal infections, some produce chronic infections with preimmunization, and some allow complete recovery and acquire immunity. T. vivax is more virulent than T. congolense, but both can cause anemia and cachexia, infection with $T$. congolense regularly results in chronic disease, while in high percentage of animals infected with T. vivax there is sudden death due to salmonellosis or other infections [14]. There are no pathognomonic signs of trypanosomiasis. Chronically affected animals are cachectic and have a rough hair coat, and there is increased clear fluid in body cavities, also abnormal enlargement lymph node is seen [15]. Identification of motile trypanosomes in the Buffy coat zone of a microhematocrit capillary tubes using dark illumination is apparently the most accurate of all diagnostic methods. Trypanosomes can be differentiated by their morphological features, manner of attachment to erythrocytes and type of motility. In endemic areas the incidence of infection by pathogenic species varies depending on factors such as the availability of mammalian reservoirs, the density of vector tsetse flies and the system of husbandry adapted, but as a rule, domestic animal and trypanosomes cannot co-exist. The pathogenic trypanosomes differ widely in virulence and apparently non-patho- genic in mammalian reservoir hosts [16].

During their life cycle in the mammalian host and in the tsetse fly vector, African trypanosomes proceed through several morphologically distinguishable stages. Infection of mammalian host is initiated by the injection of metacyclic trypanosomes present in this vector saliva [17]. Once introduced into the body of mammalian host; metacyclic trypanosomes become differentiated into rapidly proliferating (generation 4-6 hours); long, slender blood stream form parasites [18].

The life cycle of trypanosomes in tsetse involves cyclical development for varying length of time, depending on species and ambient temperature. T. vivax completes its developmental cycle in the proboscis and pharynx and can be transmitted as metacyclic trypanosomes within a few week of initial infective feed. The cycle of $T$. congolense involves the mid gut and the proboscis and the tsetse and is completed in about two weeks. That of $T$. brucie is more complex; it takes 3 or more weeks and involves the mid gut and salivary glands. Once infected, flies remain alive for 1 to 2 months [19]. For any fly, its vectorial capacity and efficiency are the highest for $T$. vivax [20]. In non-cyclical transmission, trypanosomes can be transmitted in the absence of tsetse flies (Glossina), after trypanosomes have been introduced into a herd. Biting flies such as tabanidae, stomoxynaee and hippoboscidae are capable of transmitting in their mouthparts if they feed on more than one host in a short interval. T. vivax is commonly spread by this mechanism [21].

The objectives of this study were:

1. To determine the prevalence of bovine trypanosomosis in and around nekemte surroundings.

2. To identify trypanosome species involved during study period.

\section{Materials and Methods}

\subsection{Study Areas}

Study area is located in Oromia regional state of east Wollega, in Nekemte, 330 kilometers west of Addis Ababa. The altitude ranges in these areas are from 800 to 2000 meters above sea level. The climate in the study areas can be divided into three distinct seasons: a short rainy season: between late February and early May; a longer more reliable rainy season (from June to September) and dry season (from October to May). The study areas are densely populated with approximately $80 \%$ of the total human population lives in rural areas and practices mixed farming, i.e., subsistence, rain fed cropping combined with keeping livestock are practiced.

\subsection{Study Population}

For high density of livestock population, over 74574 cattle are found in a Gutogida Woreda. These include young, adult and old animals, and also it is a total of male and female animals.

\subsection{Study Design}

A cross-sectional type of study was conducted to determine the prevalence of bovine infection with trypanosomes and to identify the prevailed species in East Wollega, in and around Nekemte surroundings from November 2010 to April 2011. 


\subsection{Sample Size}

Sample size was determined by using the method recommended by [11].

$$
\begin{gathered}
n=\text { Pexp (100-Pexp) } \\
\mathrm{SE}^{2+} \operatorname{Pexp}(100-\mathrm{Pexp}) / \mathrm{N}
\end{gathered}
$$

Where Pexp = expected prevalence;

$\mathrm{SE}=$ standard error $(0.5 \%)$;

$\mathrm{N}=$ total cattle population in and around Nekemte surroundings;

$\mathrm{n}=$ required sample.

Accordingly, the estimated sample size was 384; however, to increase the accuracy, maximum number (400) was included in the study.

\subsection{Study Tools/Procedures}

The parasitological diagnostic tests used were those described by [22]. In brief, blood was collected from an ear vein in to heparinised micro haematocrit centrifuge capillary tubes and transferred onto glass slides in order to make thin and thick blood smears. Each capillary tube was filled to its last third, sealed with the crystal sea at one end, and centrifuged immediately in a micro haematocrit centrifuge for 5 minutes at 10,000 rpm. After centrifugation, the packed cell volume (PCV) was determined. Animals with PCV $<24 \%$ were considered as anemic. The Buffy coat and the upper most layers of red blood cells in each specimen were extracted, placed onto microscope slide, and examined under phase contrast microscope with an $\times 40$ objective lens for the presence of trypanosomes. The thick and thin blood smears were stained with Giemsa and examined under a light microscope using an $\times 100$ oil immersion objective lens. At the time of sampling the information like: age, sex and body condition of each animal were recorded [23].

\subsection{Data Analysis}

Data collected during study period was recorded and entered Microsoft excel program. Statistical analysis was done using Stata version 11.0 statistical software program [24]. Statistics was used to summarize the data, statistical tests such as chi-square test, $t$ test were used to see the association between variables of study like sex, age and mean PCV.

\section{Results}

Out of 400 animals sampled, only 36 animals were found to be infected with trypanosomes. Therefore overall prevalence is found to be $36(9 \%)$. The adult animals were mostly affected groups $30(0.075 \%)$. Two species trypanosomes were identified: $T$. vivax, which is highly prevalent with the rate of $26(0.065 \%)$ and out of this it mostly affects the adult animals $(0.0575 \%)$ and $T$. congolense occurs with the low rate of $10(0.025 \%)$, out of which it commonly affects adult animals 7 (0.0175\%) and $3(0.0075)$ in young animals and absent in old animals. Female animals are commonly affected with the rate of $22(0.055 \%)$, out of which $T$. vivax is frequently occur $16(0.04 \%)$ and in male animals it occurs at lower rate of $14(0.035 \%)$, out of which $10(0.025 \%)$ are $T$. vivax and $4(0.01 \%)$ are $T$. congolense.

Maximum PCV value of the infected animals was 23 and the minimum PCV value of these animals was 16. The obtained value indicate that these animals are in anaemic condition which results from either diseased condition caused by trypanosomes or due to other predisposing factors like malnutrition, helminthosis and hemorrhagic septicaemia.

The difference in prevalence among age groups and sex categories was not statistically significant ( $\mathrm{p}>0.05)$. The mean PCV of infected animal was 19.36 and this was significantly lower $(\mathrm{p}<0.001)$ than the mean PCV of the animals that were parasitologically negative (27.54). Therefore difference of mean PCV of infected and noninfected animals were significant.

\section{Discussion}

The result of present study revealed that trypanosomosis was one of the diseases of cattle in eastern Wollega, in Nekemte areas, with overall infection rate of $9 \%$. The prevalence of trypanosomosis was lower. Table 1 in- 
Table 1. Prevalence of Trypanosome species.

\begin{tabular}{ccc}
\hline Trypanosoma species & Frequency & Percent (\%) \\
\hline T. congolense & 10 & 0.1 \\
T. vivax & 26 & 0.26 \\
Total & 36 & 0.36 \\
\hline
\end{tabular}

dicates prevalence of trypanosome species. Morphological identification of trypanosome involved in the study areas revealed that Trypanosoma vivax and Trypanosoma congolese were involved in infection. Infection due to $T$. brucei was not noted in this study; however, it is important to note that $T$. brucei are capable of invading extra vascular tissue and accumulating in loose connective tissue, which make their detection in the blood difficult [25]. Table 2 shows prevalence of trypanosome species on basis of age; in younger animals, 4 trypanosomes are identified, out of which 3 are T. congolense and 1 is T. vivax. In adult animals, 30 trypanosomes were found, out of which 7 are $T$. congolense and 23 are $T$. vivax. Table 3 indicates prevalence of trypanosome species on basis of sex; in female animals 22 trypanosomes have been found, out of which 6 are $T$. congolense and 16 are $T$. vivax. In male animals, 14 trypanosomes have been seen, out of which 4 are $T$. congolense and 10 are $T$. vivax. The higher infection rate observed in adult animals might be associated with the higher animal and tsetse contact resulting from the concentration of flies on river banks where there was green pasture and access to drinking water.

The cumulative effect of exposure to tsetse flies and new strains of trypanosomes in adult animals accounts for the observed infection rate. The reduced infection rate in older animals might be associated with the innate resistance of cattle, which increased by repeated exposure of the same population of trypanosomes in a given area. T. vivax was the potentially dominant species identified in this study with relative proportion of 26 $(0.065 \%)$, but $T$. congolense was with lower rate of $10(0.025) \%$. This is attributed to the fact that as distance from the known tsetse infestation increases, T. vivax becomes more frequent and eventually predominated [26]. The findings support this conclusion, meaning from a research conducted or survey of trypanosomes in eastern Wollega, Nekemte province determined that of 400 cattle examined at Guto Gida in the present study area, 36 were positive for trypanosomes. Among which only 10 were due to $T$. congolense while the remaining (26) were due to $T$. vivax infections. According to information available on the presence of tsetse flies on the study area and geographical location of the study site in relation to Nekemte surroundings, Glossina are present, which strongly indicates the possible advancement of the fly and the trypanosomes.

An epidemiologically important observation in this study is the infection of animals with tsetse transmitted trypanosome: trypanosome, $T$. vivax and $T$. congolense. There are some rivers, therefore; it is more likely that tsetse flies migrate up land, from their original habitat, following the river courses. The movement of tsetse away from their prime habitat when climatic conditions are not favorable in the surrounding areas has been described [27]. Furthermore, the uncontrolled movement of cattle from neighbouring areas of Nekemte with confirmed cases of $T$. congolense and $T$. vivax may have contributed to the occurrence of $T$. congolense and the maintenance of $T$. vivax.

The reproduction and development of biting flies after the end of heavy rainy seasons occurs and this increases the rate of spread of the disease. Packed cell volume (PCV) is the most reliable indicator of anemia in trypanosomes [28]. Our observation that trypanosome infections result in a significant decline in PCV is in agreement with previous findings in [29] and [16] which reported that trypanosomes caused depressed PCV levels in infected animals and other factors such as malnutrition or other disease might affect the PCV and body condition. However, it is unlikely that the impact of those additional factors differs greatly between the parasitological positive and negative animals [30]. Hence, relative comparisons between the age groups and sexes of parasitological positive and negative animals can be made. From the present study, it can thus be concluded that trypanosomosis is a major livestock disease and a potential threat in affecting the health and productivity of cattle in the economically important districts of the east Wollega, Nekemte Zone and its surroundings.

\section{Conclusion and Recommendations}

Trypanosomosis is a serious and fatal disease of all domestic and wild animals, including human beings. It causes a considerable economic loss in our countries through loss of livestock. This commonly occurs in areas associated with tsetse flies, where the disease is rapidly transmitted by Glossina species as an intermediate host. 
Table 2. Prevalence of Trypanosome species on basis of age.

\begin{tabular}{cccc}
\hline Age group & No. of animals examined & No of + ve & Prevalence (\%) \\
\hline young & 29 & 4 & 13.79 \\
Adult & 319 & 30 & 9.40 \\
Old & 52 & 2 & 4.0 \\
Total & 400 & 36 & 9.00 \\
\hline
\end{tabular}

Pearson $\operatorname{chi}^{2}(2)=2.5637, \mathrm{p}=0.278$

Table 3. Prevalence of trypanosome species on basis sex.

\begin{tabular}{cccc}
\hline Sex group & No. of animals sampled & No of + ve & Prevalence (\%) \\
\hline Female & 262 & 22 & 8.4 \\
Male & 138 & 14 & 10.14 \\
Total & 400 & 36 & 9.00 \\
\hline
\end{tabular}

Pearson $\operatorname{chi}^{2}(2)=0.3372, \mathrm{p}=0.56$.

Based on the above conclusion the following recommendations are forwarded:

- Give a prophylactic treatment while moving animals through an area with tsetse flies.

- Use of trypanotolerant breeds is recommended (sheko breeds) in Ethiopia and N'dama in the world.

- Control of tsetse flies population by insecticide spray, such as ground spray, should be applied.

Prevention of tsetse-fly by different scientific methods, such as sterile insect technique, use of accaricides, and destroying of tsetse belt, should be applied.

\section{References}

[1] FAO (2005) The Influence of Trypanosomosis on African Animal Production. Animal Zootechnica, 1-2.

[2] Murry, M. (1978) Anemia of Bovine trypanosomes. In: Losos, G.J. and Chouinard, A., Eds., Pathogenesis of trypanosomes, No 1320, IDRC, Ottawa, 121-127.

[3] Slingerbergh, J. (1992) Tsetse Control and Agricultural Development in Ethiopia. World Animals’ Review, 70-71, 30-36.

[4] Van den Ingh, T.S.G.A.M., Zwart, D., Van Miert, A.S.J.P.A.M. and Schotman, A.J.H. (1976) Clinico-Pathological and Pathomorphological Observations in Trypanosomes in Trypanosome vivax Infection in Cattle. Veterinary Parasitology, 257, 135-138. http://dx.doi.org/10.1016/0304-4017(76)90082-0

[5] Urquhart, G.M., Armour, J., Dunkan, J.L., Dunn, A.M. and Jennings, F.W. (1996) Veterinary Parasitology. 2nd Edition, Long English Language Society, 212-216.

[6] Fiennes, R.N.T. (1982) The Cattle trypanosomes a Cryptic to Causes of Parasites in Association with Secondary Stage of Disease. Br Vet. F, 108, 298- 305.

[7] Soulsby, E.J.L. (1982) Helminthes, Arthropods and Protozoa of Domesticated Animals. 7th Edition, Bailniere Tindall, London, 527.

[8] Radostits, O., Gay, M., Hinchcliff, C.C. and Constable, P.D. (2007) Veterinary Medicine. A Text Book of Disease of Cattle, Sheep, Pigs and Goats. 10th Edition, Saunders Ltd., 1531-1540.

[9] Kobayashi, A., Tizard, I.R. and Woo, P.T. (1976) Studies on the Anemia in Experimental African Trypanosomiasis.II the Pathogenesis of the Anemia in Calves Infected with Trypanosoma congolense. The American Journal of Tropical Medicine and Hygiene, 25, 401-406.

[10] Jordan, A.M. (1988) Trypanosomiasis Control and African Rural Development. Longman, London, 233.

[11] MacLennan, K.J.R. (1983) Tsetse-Transmitted Trypanosomiasis in Relation to the Rural Economy in Africa. Part I: Tsetse Infestation. FAO Animal Production and Health Paper, 37, 48-63.

[12] Rocha, G., Martins, A., Gama, G., Brando, F. and Autoguia, J. (2004) Possible Cases of Sexual and Congenital Transmission of Sleeping Sickness. The Lancet, 363, 247. 
[13] Packchanian, A. (1974) Studies on Trypanosome Gambiense in Various Species of Experimental Animals. Texas Reports on Biology \& Medicine, 22, 707-715.

[14] Jubb, K.V.F., Kennedy, P.C. and Palmer, N. (2007) Pathology of Domestic Animals: Volume 3. 5th Edition, Saunders Ltd., New York, Oxford, Philadelphia, St. Luis, Sydney and Toronto, 274-276.

[15] Hunter, A.G. and Luckins, A.G. (1990) Trypanosomosis. In: Sewell, M.M.H. and Brocklesby, D.W., Eds., Handbook on Animal Diseases in the Tropics, Fourth Edition, Bailiere Tindall, London, 204-226.

[16] Losos, G.J. and Ikede, B.O. (1972) Review of the Disease in Domestic and Laboratory Animals Caused by Trypanosoma congolense, T. vivax, T. brucei, Trypanosoma rhodesiense and Trypanosoma gambiense. Veterinary Pathology, 9, 1-79. http://dx.doi.org/10.1177/030098587200901s01

[17] Leach, T.M. (1973) African Trypanosomiasis. Advances in Veterinary Science \& Comparative Medicine, 17, 119-162.

[18] Desquesnes, M. (2004) Livestock Trypanosomes and Their Vectors in Latin America. OIE (World Organization for Animal Health), Paris, 256.

[19] Ikede, B.O. (1986) African Trypanosomes. In: Honigberg, B.M., Ed., Mechanisms of Pathogenicity among Protozoa, Bailiere Tindall, London, 363-378.

[20] Putt, S.N.H., Shaw, A.P.M., Woods, A.J., Tyler, L. and James, A.D. (1988) Veterinary Epidemiology and Economics in Africa: A Manual for Use in the Design and Appraisal of Livestock Health Policy. ILCA, Addis Ababa, 231.

[21] Paris, J., Murray, M. and McOdimba, F. (1982) A Comparative Evaluation of the Parasitological Techniques Currently Available for the Diagnosis of the African Trypanosomes in the Cattle. Acta Tropica, 39, 307-316.

[22] Nicholson, M.J. and Butterworth, M.H. (1986) A Guide to Condition Scoring of Zebu Cattle. ILCA, Addis Ababa, 235.

[23] Stata Version 11.0 (2009) Stata Corporation 4905 Luke Way Drive College Station, Texas 77845, USA.

[24] Uilenberg, G. (1998) A Field Guide for the Diagnosis, Treatment and Prevention of African Animal Trypanosomosis. FAO Corporate Document Repository. Food and Agriculture Organization of the United Nations, Rome.

[25] Roeder, P.L., Scott, J.M. and Pegram, R.G. (1984) Acute Trypanosoma vivax Infection of Ethiopia Cattle in the Apparent Absence of Tsetse. Tropical Animal Health and Production, 16, 141-147. http://dx.doi.org/10.1007/BF02252779

[26] Uilenberg, G. (1998) A Field Guide for the Diagnosis, Treatment and Prevention of African Animal Trypanosomosis. FAO Corporate Document Repository.

[27] Van den Bossche, P., Shumba, W. and Makhambera, P. (2000) The Distribution and Epidemiology of Trypanosomes in Malaysia. Veterinary Parasitology, 88, 163-176.

[28] Morrison, W.I., Murray, M. and McIntyre, W.I. (1981) Bovine Trypanosomosis. In: Ristic, M. and McIntyre, I., Eds., Diseases of Cattle in the Tropics: Economic and Zoonotic Relevance, Volume 6, Martinus Nijhoff Publishers, The Hague, 486-488.

[29] Anosa, V.O. and Obi, T.U. (1980) Haematological Studies on Domestic Animals in Nigeria. III. The Effects of Age, Breed and Haemoglobin Type on Bovine Haematology and Anaemia. Zentralblatt fur Veterinarmedizin, 27B, 773-788.

[30] Anosa, V.O., Logan-Henfrey, L.L. and Wells, C.W. (1999) The Role of the Bone Marrow in Bovine Trypanotolerance II. Macrophage Function in Trypanosoma congolense-Infected Cattle. Comparative Haematology International, 9, 208-218. 